criticize the organization of the symposium, but only to comment that the absence of these viewpoints necessarily makes the volumes less useful as a summary of current thinking on photosynthesis.

In any case, is it worthwhile to review current thinking on photosynthesis in book form, rather than to rely on publication in journals? This is especially important in view of the interval of 18 months between the conference and the appearance of the book in Britain. This lag means that the book is inevitably dated by the time it appears; as an example, Arnon's heretical views have subsequently undergone schism ${ }^{1}$.

By putting a wide range of photosynthetic research into these three volumes, it is possible that some crossfertilization will occur between different approaches to photosynthesis. It may be argued that the profitable cross-fertilization occurs laterally, with, for example, physical chemistry, mitochondriology and micrometeorology, rather than between, say, the physico-chemical and ecological approaches to photosynthesis itself. Still, it is as well to leave the doors open. The specialist in one aspect of photosynthesis can find, in these volumes, a summary of the conclusions reached by contributors to other specialized sections, and provides entry to current research in other fields. These volumes thus develop their theme at two levels of complexity, and are the more useful for that.

Cross-fertilization can be aided by the excellent index, repested in each volume, which makes the following of one particular point (for example, the mode of action of an inhibitor) easier than would the presentation of the same papers in a variety of journals, abstracting journals notwithstending. One important feature often found in symposium reports, but lacking in this instance, is a record of the verbal discussion which followed each paper. These discussions often place the work in a wider context, as well as pointing out directly relevent technical or conceptual points. One can but hope that at least some of the points raised in discussion have been incorporated into the papers as printed in these volumes.

In those sections in which I have greatest knowledge of the literature, it would seem thet about half of the papers in these volumes are substantially different from papers which have appeared in journals. The overlap, and the importance of the time lag in publication, is greater for papers on electron transport and energy coupling than for, for example, photorespiration and photophosphorylation in vivo. To this extent the volumes are more useful as a unique source in these less central topics in photosynthetic research. Unfortunately, there is no way of determining how much of the interesting material exclusively found here would have appeared elsewhere if these volumes had not been published.

Assuming that this material would have been published elsewhere, are these books more useful than journal publications, together with some literature survey (such as the one in Photochemistry and Photobiology, which has about the same total time lag, that is publication of paper and appearance in the literature survey, as does the appearance of the papers in this book) ? It is convenient to have the papers collected together in bound form, together with the index and interpretative summaries, although more wide-ranging summaries occur in other review articles. It is useful as a source for research workers, university teachers and (possibly) advanced undergraduates. Few will be able to afford it, unless they took advantage of the pre-publication subscription offer; it must undoubtedly be available in university and research institute libraries.

Let it not be thought that I am demning with faint praise; within the limitations of the bound book format, Dr Metzner has done very well in assembling the very large number of papers into three such attractive and well produced volumes.

JOHN RAVEN

Knaff, D. B., and Arnon, D. I., Proc. VS Nat. Aoud. Sot., 64, 715 (1969).

\section{RADIOLOGICAL RECIPES}

\section{Manual on Radiation Dosimetry}

Edited by Niels W. Holm and Roger J. Berry. Pp. xvi+ 450. (Dekker: New York, April 1970.) \$24.50; 2338 .

RADIATION dosimetry is an art which, like cookery, depends on well tried and tested recipes carefully prepared and elegantly presented. This rather expensive manual provides twenty step-by-step recipes supported by reviews of the principles on which they are based. But who will use it ? The editors give a clue by selecting "areas" of application-radiation protection in hospitals, radiation therapy and the industrial use of cobalt-60 sources and accelerators.

This manual lacks adequate cross-referencing and the editors do not impose consistent terminology on the authors of individual chapters. Their own introduction omits the recommendations of the International Commission on Radiological Units and Measurements ${ }^{1}$ which must surely be taken as the authority on terminology. Readers would do well to turn to W. L. MoLaughlin's chapter on "Films, Dyes and Photographic Systems" in which they will find a well referenced survey of the "jungle" of hundreds of available dosimetry systems.

T. E. Burlin's treatment of dosimeter response has a few small and obvious errors, but this is a clear and essily read chapter which could be usefully expanded to cover detectors other than ionization chambers. This chapter and that on calorimetry are not supported by recipes, although these fundamental dosimetry methods require at least as much skill as the more obvious cookery book methods. Abundant recipes and advice are offered on aqueous dosimeters, leaving little comfort for those readers interested in non-aqueous systems (such as nitrous oxide), which may be better for the measurement of high total dose.

The editors were fortunate to have contributions from J. R. Cameron and F. M. Attix on "Radiophotoluminescent and Thermoluminescent Dosimetry"-a brief reference to thermally stimulated electron emission would have completed a thorough survey of this important dosimetry field.

The use of anatomical phantoms in dosimetry deserves more than the brief mention by L. H. Lanzl, who describes the application of dosimetry to medical radiation therapy. Future editions would benefit from an examination of the tissue equivalence of phantom material and available phantom systems.

The recipes in this manual, taken with the principles, are suitable for users of gamma sources, particularly cobalt-60 and accelerators. It is of lesser value to reactor health physicists who will find little reference to neutrons and none to mixed field dosimetry. Future editions will be improved by more severe editing, and a sound system of terminology would raise this book of favourite recipes to the Escoffier class. J. R. A. LAKEY

1 Radiation Quantities and $D$ nits, Report $10 \mathrm{a}$ of the Internatlonal Commisgion on Radiological Units and Measurements. (NBS Fand
National Burean of Standards, Washington DC, 1962.)

\section{PRINCIPLES OF BAND THEORY}

\section{Band Theory of Metals}

The Elements. By Simon L. Altmann. Pp. xiv +250 . (Pergamon: Oxford and New York, June 1970.) 50s (\$6.75) board; 358 (\$4.75) flexi-cover.

THE theory of the solid state is dominated by Bloch's theorem: an electron in a regular crystal lattice must have wave-like quantum states, each of a specific wave vector. The proof of this theorem, and an examination of its consequences, first in one-dimension, then in more complicated three-dimensionel lattices, is indispensable to any understanding of the modern theory of metals and semiconductors. The mathematics needed is not very 